\title{
Constraining chemical migration and partitioning in laser-heating diamond anvil cell studies by combining experiments \& finite element modelling.
}

NICOLAS JAISLE ${ }^{1}$, DAVID CEBRON ${ }^{2}$, ANGELIKA

DOROTHEA ROSA ${ }^{3}$ AND GUILLAUME MORARD ${ }^{4}$

${ }^{1}$ ISTerre CNRS UGA

${ }^{2}$ ISTerre-CNRS-UGA

${ }^{3}$ European Synchrotron Radiation Facility

${ }^{4}$ Université Grenoble Alpes-CNRS-IRD-IFSTTAR-ISTerre

Presenting Author: nicolas.jaisle@univ-grenoble-alpes.fr

The physical properties of planetary cores are of fundamental importance to understand the dynamics of planetary interiors. Studying experimentally such properties, including phase diagrams, thermal conductivity and elasticity remains however challenging. A major difficulty is the reproduction of relevant core conditions, i.e., $6000 \mathrm{~K}$ and $360 \mathrm{GPa}$ for the Earth.

The laser-heated diamond Anvil Cell (LH-DAC) is a high P/T device that allows reaching those conditions and which can be easily combined with X-Ray diagnostics for in-situ structure determinations. High and homogenous temperatures in these micrometric samples are however only achieved in the very small focal spot of the YAG lasers (20 microns). This leads to thermal gradients in the sample that induce chemical migrations which are difficult to disentangle from chemical partitioning. Due to these difficulties, most studies have been focused on the determination of eutectic melting and not liquidus temperatures for binary systems. In addition, temperature measurements in these devices rely on spectroradiometry and are thus restricted to the sample surface.

To circumvent this problematic we developed an original approach based on finite element (FE) calculations to determine thermal gradients and chemical migration conditions in the sample during LHDAC experiments. FE calculations assess temperature gradients in a similar way to earlier studies upon XRay heating (i.e., Meza-Galvez et al. 2020), while those aiming to reproduce fusion and convection phenomena are, among other, based on the Phase Field method. Finally, the model includes as well the effect of thermal diffusion of elements (Soret effect) as described by Zhang et al. 1989 \& Sinmyo et al. 2010.

In a first step, and as a proof of concept, we applied these methods to pure iron, the main constituent of planetary cores. In a second step, we studied $\mathrm{FeSiO}$ ternary system that has attracted high attention as both $\mathrm{Si}$ and $\mathrm{O}$ are two potential light elements constituting of the Earth's \& exoplanets cores (Hirose et al. 2017). The results of FE models will serve as diagnosis to interpret chemical migration in terms of elemental partitioning in future static compression studies, such as in the experiment planned at the Eu-XFEL in November 2021. 